\title{
Relationship between anxiety, depression and quality of life in medical student with polycystic ovary syndrome
}

\author{
Rita Saxena $^{1}$, Pooja Singh ${ }^{1 *}$, Anjana Verma ${ }^{1}$, Manu Sharma ${ }^{2}$
}

\begin{abstract}
${ }^{1}$ Department of Obstetrics and Gynecology, ${ }^{2}$ Department of Psychiatry, Geetanjali Medical College and Hospital, Udaipur, Rajasthan, India
\end{abstract}

Received: 15 November 2021

Revised: 27 November 2021

Accepted: 30 November 2021

\section{*Correspondence:}

Dr. Pooja Singh,

E-mail: pooja26june6@yahoo.com

Copyright: () the author(s), publisher and licensee Medip Academy. This is an open-access article distributed under the terms of the Creative Commons Attribution Non-Commercial License, which permits unrestricted non-commercial use, distribution, and reproduction in any medium, provided the original work is properly cited.

\begin{abstract}
Background: Polycystic ovary syndrome (PCOS) is the most common endocrine disorder affecting 5-10\% of women worldwide. It has clinical, hormonal and psychological manifestation like anxiety, depression and low self-esteem. Aim was to evaluate prevalence of anxiety, depression, and quality of life in medical students with PCOS. Objectives were to study the prevalence of anxiety, depression among medical students suffering from PCOS; and to evaluate quality of life (QoL) in students with PCOS and its association with anxiety, depression and quality of life.

Methods: The cross sectional study was conducted among 70 medical students at Geetanjali Medical College and Hospital, Udaipur, Rajasthan, from February 2019 - June 2020. Students were screened and selected as per the Rotterdam's 2003 criteria after informed consent. Mental health assessment done by using Hamilton depression rating scale for depression, Hamilton anxiety rating scale for anxiety, and using 36-item short form survey for QoL.

Results: Twenty five (35.7\%) students suffered from anxiety while 20 (28.5\%) had depressive disorders. Forty five $(64 \%)$ patient both anxiety and depression. These students had significant psychological morbidity and poor QoL.

Conclusions: Psychological morbidity with PCOS is undertreated and need to be addressed and treated in time so as to turn these students into a responsible happy adult.
\end{abstract}

Keywords: PCOS, Anxiety, Depression, Quality of life

\section{INTRODUCTION}

Polycystic ovary syndrome (PCOS) is an endocrine disorder common among women of reproductive age. ${ }^{1}$ PCOS is a heterogeneous disorder of uncertain cause ${ }^{2}$ and it may present clinically with menstrual irregularities, signs of androgen excess and obesity. The clinical implications of PCOS are reproductive (infertility, hyperandrogenism, hirsutism), metabolic (insulin resistance, impaired glucose tolerance, type 2 diabetes mellitus, increased risk of cardiovascular events) and psychological features (increased anxiety, depression, and low self-esteem). ${ }^{3} \mathrm{~A}$ multi-faceted approach is required in the treatment of PCOS that includes lifestyle modification, monitoring of metabolic complications and psychological support. ${ }^{5-7}$

Previous studies have reported psychological problems such as anxiety, depression and body image concerns. ${ }^{8}$ Consequently, PCOS is likely to have an impact on the quality of life (QoL). ${ }^{8}$ Studies during early adulthood are likely to improve the understanding of psychological issues and QoL affecting persons with PCOS. ${ }^{9-11}$

There is a paucity of literature from India addressing the psychological morbidity in medical undergraduates and post-graduates with PCOS. Given this background, the present study was conducted with the objectives of 
determining the relationship between anxiety, depression and QoL in health science students with PCOS.

\section{Aim}

Aim of the study was to assess prevalence of anxiety, depression and quality of life in place of health care workers write medical students.

\section{Objectives}

The objectives of the research were to study the prevalence of anxiety, depression among women suffering from PCOS and to evaluate quality of life in students with PCOS and its association with body satisfaction, anxiety and depression.

\section{METHODS}

The present cross-sectional study was conducted on female medical undergraduates and post-graduates, of a tertiary care teaching hospital in Udaipur, Rajasthan, India. The students were invited to participate in the present study through announcements in their classes and departments. The institute ethics committee approved the study protocol and written informed consent was obtained from the participants. Each consenting participant was provided with a pre-designed performa to collect sociodemographic, general and gynaecological health information. Students with a history of psychoactive substance use in the preceding 6 months (other than nicotine), major psychiatric and other medical disorders were excluded. The diagnosis of PCOS was based on the Rotterdam 2003 criteria. Of the 78 participant were diagnosed with PCOS, eight of them were excluded as their data forms incomplete. Thus, 70 students diagnosed with PCOS constituted the study population. The students underwent evaluation of depression, anxiety and QoL using the Hamilton depression rating scale (HDRS), Hamilton anxiety rating scale (HARS) and 36-item short form survey (SF-36), respectively. A brief description of the study tool is given below.

\section{Hamilton depression rating scale (HDRS)}

It is a multiple item questionnaire is used to rate the severity of depressive symptoms. The theoretical score range of the scale is from 0 to 52 . A score of $0-7$ is generally accepted to be within the normal range (or the patient is in the clinical remission phase), while a score of 20 or higher indicating at least moderate severity of the depression. $^{12}$

\section{Hamilton anxiety rating scale (HARS)}

It was one of the first rating scales developed to measure the severity of anxiety symptoms, and is still widely used today in both clinical and research settings. The scale consists of 14 items, each defined by a series of symptoms, and measures both psychic anxiety (mental agitation and psychological distress) and somatic anxiety. Each item is scored on a scale of 0 (not present) to 4 (severe), with a total score range of $0-56$, where $<17$ indicates mild severity, 18-24 mild to moderate severity and 25-30 moderate to severe. ${ }^{13}$

\section{6-item short form survey (SF-36)}

It is considered to be a measure of health related QoL (HRQoL) constructed to be suitable for use by anyone, irrespective of demographics or disease, and contains 36 items. It measures physical and mental health based on 8 health concepts: physical and social functioning, role limitations due to physical and emotional problems, mental health, vitality, bodily pain, and general health perception. ${ }^{14}$

\section{Data analysis}

All data collected using HDRS, HARS and SF-36 questionnaire were recorded in a statistical package for the social sciences (SPSS) spreadsheet after coding. The categorical data and continuous data were expressed as percentages and mean ( \pm standard deviation), respectively. Test of association between various determinants with risk/presence of PCOS among students was done using $\mathrm{t}$ test. $\mathrm{P} \leq 0.05$ was taken as the cut-off for significant association.

\section{RESULTS}

Of the 78 participants who took part in this study, 8 were excluded as their forms were incomplete. Thus, a total of 70 females in the age group of 18-45 years were studied. The mean age of the sample was $25.65 \pm 7.60$ years (Table 1). Around $17 \%$ of the students with PCOS were classified as overweight and $5 \%$ were obese as per their body mass index (BMI).

Table 1: Distribution of age and body mass index (BMI) of the study participants $(n=70)$.

\begin{tabular}{|lc|}
\hline Variables & $\mathbf{N}(\%)$ \\
\hline Age (years) & \\
\hline $18-20$ & $27(38.5)$ \\
\hline $21-30$ & $36(51.5)$ \\
\hline$>30$ & $7(10)$ \\
\hline BMI & $11(15.7)$ \\
\hline Underweight & $37(52.8)$ \\
\hline Normal & $17(24.2)$ \\
\hline Overweight & $5(7.1)$ \\
\hline Obese & \\
\hline
\end{tabular}

\section{Prevalence of anxiety and depression}

Of the 70 females studied, 25 were found to be suffering from anxiety disorders, while 20 were found to be suffering from depressive disorders. Thus, the prevalence of anxiety disorders in our study sample was $35.7 \%$, and 
the prevalence of depressive disorders was $28.5 \%$. And a total of 25 patients, that is, $35.7 \%$, had neither of the two.

The Hamilton rating scales for anxiety and depression were used to rate the severity of the respective conditions among those, in whom they were present (Table 2).

The $t$ value is 5.94342 . The $p$ value is $<0.00001$. The result is significant at $\mathrm{p}<0.05$.Thus, patients with psychiatric morbidity had a significantly lower QoL. But irrespective of the above comparison QoL of female suffering from PCOS is affected with and without psychiatric morbidities.
Table 2: Distribution of anxiety and depressive symptoms in the study participants $(n=70)$.

\begin{tabular}{|ll|}
\hline Anxiety and depressive symptoms & Percentage \\
\hline Anxiety & \\
\hline Mild & 32 \\
\hline Moderate & 40 \\
\hline Severe & 28 \\
\hline Depression & \\
\hline Mild & 45 \\
\hline Moderate & 30 \\
\hline Severe & 25 \\
\hline
\end{tabular}

Table 3: Relationship between quality of life and psychological morbidity (anxiety and depressive symptoms) in the study population.

\begin{tabular}{|lllll|}
\hline Quality of life & $\begin{array}{l}\text { PCOS with psychological } \\
\text { morbidity }(\mathbf{n}=\mathbf{4 5})\end{array}$ & $\begin{array}{l}\text { PCOS without psychological } \\
\text { morbidity }(\mathbf{n}=\mathbf{2 5})\end{array}$ & $\mathbf{t}$ & $\mathbf{P}$ \\
\hline Mean ( $\mathbf{n S D})$ & $61.57 \pm 1.55$ & $49.28 \pm 2.73$ & 5.943 & $<0.0001$ \\
\hline
\end{tabular}

\section{DISCUSSION}

The overall prevalence of psychological morbidity in the present study was $64.2 \%$. Comparisons across studies are difficult due to vast differences in methodology.

Previous studies have found that women with PCOS tend to experience mildly elevated anxiety and depression. ${ }^{15}$ This is consistent with findings of the present study. The reasons for the higher prevalence of anxiety and depression in PCOS are complex. Emotional distress due to the symptoms and their psycho-social consequences could be contributory factors to the psychological morbidity. ${ }^{16}$ A study which used a structured clinical interview found an overall prevalence of psychiatric morbidity to be $52.7 \% .{ }^{17}$ The findings of the present study are consistent with previous investigations. ${ }^{18}$

In the present study, the psychological domain of SF-36 had the lowest score and psychiatric morbidity was significantly associated with lower QoL. Several studies have reported that women with PCOS who suffer from anxiety and depression have a lower QoL. ${ }^{19.20,21}$ Some authors suggest that psychological morbidity can impact on physical (e.g. eating and sleeping patterns), psychological (e.g. motivation and feelings of worthlessness), and social factors (e.g. relationships with others).

Further multicentric studies are needed to elucidate the complex interaction between psychological morbidity in persons with PCOS. Effective symptom management of PCOS is likely to be improved if existing anxiety and depression are adequately treated.

The present study was a modest preliminary attempt to survey anxiety, depression and QoL in medical students with PCOS.

\section{Limitations}

The present study is encumbered by certain limitations. A small sample size, cross-sectional design and possible selfreport bias limits the generalization of the study findings. The relationship between the SF-36 sub-scales and psychological morbidity was not evaluated. Also, the authors have not taken into account the relationship of other factors such as living away from family, stressors associated with the medical profession, body image and self-esteem assessment, and peer/workplace relationships, which are associated with perceived QoL. Despite its limitations, the study confirms that anxiety and depression is associated with lower QoL in persons with PCOS. Therefore, routine screening for psychological morbidity in women with PCOS assumes clinical significance.

\section{CONCLUSION}

Around $17 \%$ of the medical students with PCOS were classified as overweight and $5 \%$ were obese. The majority of the participants reported moderate anxiety and mild depressive symptoms. The students with PCOS and psychological morbidity (anxiety and depressive symptoms) had a lower QoL compared to their counterparts without psychological morbidity. The problem that starts with the psychological morbidity gets further compounded by physical factors like eating/sleeping factor along with social factors like interpersonal relationship.

Psychological morbidity is often underrated and undertreated, therefore it calls for addressing these problems associated with PCOS in a time frame, shaping them into responsible and happy smiling adults.

Hence it calls for a "broader base" study to get a clearer picture of the problem focusing on another significant 
aspect that is "quality of life" as subjects to undergo various shades of anxiety and depression.

\section{Funding: No funding sources}

Conflict of interest: None declared

Ethical approval: The study was approved by the Institutional Ethics Committee

\section{REFERENCES}

1. Dargham SR, Ahmed L, Kilpatrick ES, Atkin SL. The prevalence and metabolic characteristics of polycystic ovary syndrome in the Qatari population. PloS One. 2017; 12:e0181467.

2. Matzke A. The Underdiagnosis of Polycystic Ovarian Syndrome in Normal Weight Adolescent Females [Dissertation]. Minneapolis: St. Catherine University. 2011.

3. Joseph N, Reddy AG, Joy D, Patel V, Santhosh P, Das $\mathrm{S}$, et al. Study on the proportion and determinants of polycystic ovarian syndrome among health sciences students in South India. J Nat Sc Biol Med. 2016;7:166-72.

4. Rotterdam ESHRE/ASRM-Sponsored PCOS Consensus Workshop Group. Revised 2003 consensus on diagnostic criteria and long-term health risks related to polycystic ovary syndrome. Fertil Steril. 2004;81(1):19-25.

5. Teede HJ, Misso ML, Deeks AA, Moran LJ, Stuckey BG, Wong JL, et al. Assessment and management of polycystic ovary syndrome: Summary of an evidencebased guideline. Med J Aust. 2011;195:65-112.

6. Alvarez-Blasco F, Botella-Carretero JI, San Millán JL, Escobar-Morreale HF. Prevalence and characteristics of the polycystic ovary syndrome in overweight and obese women. Arch Intern Med. 2006;166:2081-6.

7. Chen TH, Lu RB, Chang AJ, Chu DM, Chou KR: The evaluation of cognitive-behavioral group therapy on patient depression and self-esteem. Arch Psychiatr Nurs. 2006;20:3-11.

8. Deeks AA, Gibson-Helm ME, Teede HJ. Anxiety and depression in polycystic ovary syndrome: a comprehensive investigation. Fertil Steril. 2010;93:2421-3.

9. Michelmore KF. Polycystic ovary syndrome in adolescence and early adulthood. Hum Fertil (Camb). 2000;3:96-100.

10. Coffey S, Bano G, Mason HD: Health-related quality of life in women with polycystic ovary syndrome: a comparison with the general population using the Polycystic Ovary Syndrome Questionnaire (PCOSQ) and the Short Form-36 (SF-36). Gynecol Endocrinol. 2006;22:80-6.
11. Murri M, Luque-Ramírez M, Insenser M, OjedaOjeda M, Escobar-Morreale HF. "Circulating markers of oxidative stress and polycystic ovary syndrome (PCOS): a systematic review and meta-analysis". Hum Reprod Update. 2013;19(3):268-88.

12. Hamilton M. A rating scale for depression. J Neurol Neurosurg Psychiatry. 1960;23:56-62.

13. Hamilton M. The assessment of anxiety states by rating. Br J Med Psychol. 1959;32:50-5.

14. Lins L, Carvalho FM. SF-36 total score as a single measure of health-related quality of life: Scoping review. SAGE Open Med. 2016;4:2050312116671725.

15. Barry JA, Kuczmierczyk AR, Hardiman PJ. Anxiety and depression in polycystic ovary syndrome: A systematic review and meta-analysis. Hum Reprod. 2011;26:2442-51.

16. Veltman-Verhulst SM, Boivin J, Eijkemans MJ, Fauser BJ. Emotional distress is a common risk in women with polycystic ovary syndrome: A systematic review and meta-analysis of 28 studies. Hum Reprod Update. 2012;18:638-51.

17. Hussain A, Chandel RK, Ganie MA, Dar MA, Rather YH, Wani ZA, et al. Prevalence of psychiatric disorders in patients with a diagnosis of polycystic ovary syndrome in Kashmir. Indian $\mathbf{J}$ Psychol Med. 2015;37:66-70.

18. Cooney LG, Lee I, Sammel MD, Dokras A. High prevalence of moderate and severe depressive and anxiety symptoms in polycystic ovary syndrome: A systematic review and meta-analysis. Hum Reprod. 2017;32:1075-91.

19. Deeks AA, Gibson-Helm ME, Paul E, Teede HJ. Is having polycystic ovary syndrome a predictor of poor psychological function including anxiety and depression? Hum Reprod. 2011;26:1399-407.

20. Sayyah-Melli M, Alizadeh M, Pourafkary N, Ouladsahebmadarek E, Jafari-Shobeiri M, Abbassi J, et al. Psychosocial factors associated with polycystic ovary syndrome: A Case control study. J Caring Sci. 2015;4:225-31.

21. Barnard L, Ferriday D, Guenther N, Strauss B, Balen $\mathrm{AH}$, Dye L, et al. Quality of life and psychological well-being in polycystic ovary syndrome. Hum Reprod. 2007;22:2279-86.

Cite this article as: Saxena R, Singh P, Verma A, Sharma M. Relationship between anxiety, depression and quality of life in medical student with polycystic ovary syndrome. Int J Reprod Contracept Obstet Gynecol 2022;11:35-8. 